\title{
¿La condicionalidad como nuevo paradigma de política social en América Latina?*
}

\author{
Brenda Brown y Pablo Pérez ${ }^{* *}$
}

\section{Resumen}

En las últimas décadas se ha generalizado un proceso de reconfiguración de la protección social -workfare- que consiste en una nueva forma de gestionar los riesgos sociales que promueve el autoabastecimiento, el individualismo y la autorresponsabilidad. Estas modificaciones tuvieron su correlato en la implementación de políticas sociales y de empleo basada en necesidades perentorias y demostrables que requieren algún tipo de contraprestación. Este artículo tiene como objetivo analizar qué características adopta en América Latina esta reconfiguración del sistema de protección social haciendo énfasis en los Programas de Transferencias Monetarias Condicionadas (PTMC), cuál es la relación entre la reconfiguración del sistema de protección social y los PTMC, qué diagnóstico está detrás del consenso en la aplicación de estos programas y si todos los PTMC apuntan a un mismo

\footnotetext{
* Artículo recibido el 22 de febrero de 2016. Aceptado el 10 de julio de 2016.
}

** Brenda Brown (brenbrown87@gmail.com); Pablo Pérez (paperez@isis.unlp.edu.ar) 
De Prácticas y discursos/ Universidad Nacional del Nordeste/ Centro de Estudios Sociales

problema social. Estos son los interrogantes que guían el presente artículo.

\section{Palabras clave}

Protección social, workfare, programas de transferencias monetarias condicionadas.

\section{Abstract}

In recent decades there has been widespread a process of reconfiguration of social protection (workfare) which is a new way to manage social risks that promotes self-sufficiency, individualism and self-reliance. These changes were mirrored in the implementation of social and labor market policies which supply monetary benefits as long as recipients can demonstrate that they have met certain conditions. This article aims to analyze what features adopted in Latin America this reconfiguration of social protection system with emphasis on Conditional Cash Transfer Program (CCTP). What is the relationship between the reconfiguration of social protection system and CCTP? Which is the diagnosis behind the consensus in implementing this kind of programs? Do all CCTP point to the same social problem? These questions guide this article.

\section{Keywords}

Social protection-workfare-Conditional Cash Transfer Program.

\section{Introducción}

El comienzo de siglo se caracterizó en América Latina por la llegada al gobierno de propuestas que cuestionaron las ideas neoliberales de los años noventa. En líneas generales se puede observar que se promovió el crecimiento del producto y el empleo 
De Prácticas y discursos/ Universidad Nacional del Nordeste/ Centro de Estudios Sociales

mejorando los indicadores económicos y sociales agregados. No obstante, persisten problemáticas socioeconómicas que afectan a una parte importante de la población: desempleo, precariedad e informalidad laboral, pobreza e indigencia.

La década de los 90 se caracterizó por una agudización de estos problemas sociolaborales y por la implementación de un conjunto de políticas de empleo y sociales, en su mayoría promocionadas desde los organismos internacionales de crédito, que tendieron a requerir algún tipo de contraprestación por parte de los beneficiarios para acceder a las mismas. Ya en el nuevo siglo, la persistencia de estas problemáticas -pese a las mejoras experimentadas- condujo a un incremento significativo de este tipo de programas en toda la región. Dentro de estas políticas se destacan, por su cobertura y extensión geográfica, los programas de transferencias monetarias condicionadas (PTMC), cuyo principal objetivo es reducir la pobreza por ingresos, no solo a corto plazo -mediante un beneficio monetario- sino también a mediano plazo vía el incremento del capital humano. Estos programas han sido implementados en 16 países de América Latina y alcanzan a 70 millones de personas (Valencia Lomelí, 2008).

Lavinas (2013) vincula la proliferación de este tipo de programas a la idea de "matar varios pájaros de un tiro". Es decir que con un mismo instrumento de política se busca resolver varios problemas sociales: 1) el combate a la pobreza por ingresos, 2) el desincentivo laboral -mediante la activación de las políticas sociales- y 3) la desigualdad en el acceso a beneficios sociales entre trabajadores formales y aquellos precarios o informales.

La implementación de los PTMC forma parte de una reconfiguración del sistema de protección social a nivel mundial que comenzó a mediados de los años setenta y que se caracterizó por fomentar una nueva forma de gestionar los riesgos sociales, cuyo objetivo es promover el autoabastecimiento, el individualismo y la autoresponsabilidad en la cobertura de riesgos de la población. En este sentido, este trabajo tiene como finalidad principal analizar las características que adopta en América Latina esta nueva configuración del sistema de protección social. ¿Cuál es la relación entre la reconfiguración de la protección social y los PTMC? ¿Qué diagnóstico está detrás del consenso en la aplicación de este tipo de programas? ¿Todos los PTMC apuntan a un 
De Prácticas y discursos/ Universidad Nacional del Nordeste/ Centro de Estudios Sociales

mismo problema social? Estos son algunos de los interrogantes sobre los que buscamos debatir conceptualmente en el presente artículo.

El mismo se articula en cuatro partes: la primera señala cuáles son las características que adopta el nuevo sistema de protección social a nivel mundial, especificando diferentes formas en que los países reconocen el derecho a la protección contra los riesgos sociales. También se plantea qué características adopta esta nueva configuración de la protección social en América Latina. Luego, en un segundo apartado, se analiza el diagnóstico que hay detrás de los PTMC y se discute el porqué de su consenso en América Latina. En un tercer apartado, buscamos demostrar que pese a cierta caracterización común de los programas sociales en América Latina, se puede distinguir cierta heterogeneidad que clasificamos en tres tipos de Programas de Transferencias Monetarias Condicionadas y se discuten algunas de sus particularidades. Finalmente, reflexionamos sobre los resultados alcanzados por los PTMC y se presentan otras alternativas que se vienen discutiendo en la región para mejorar la situación social de gran parte de la población en América Latina.

\section{Riesgos, derechos y condiciones para la protección social. Una nueva configuración de la protección de los riesgos sociales en América Latina}

Todos los países capitalistas avanzados reconocen alguna forma de derecho social a la protección contra los riesgos sociales básicos: desempleo, invalidez, enfermedad y vejez; derechos que casi nunca son incondicionales, dado que para percibir una prestación, los demandantes tienen que satisfacer justamente la condición de estar enfermos, ser discapacitados o estar desempleados. En este sentido, es importante hacer una distinción entre el concepto de condicionalidad y el de contraprestación. Las condicionalidades se vinculan a ciertas características de los beneficiarios: ser pobres, desempleados, informales, etc., mientras que las contraprestaciones hacen referencia a actividades que estos tienen que realizar como contrapartida del subsidio recibido. La condicionalidad suele, a su vez, estar relacionada al tipo de convenio que la sociedad establece con la seguridad social. 
De Prácticas y discursos/ Universidad Nacional del Nordeste/ Centro de Estudios Sociales

Es clásica la distinción, en las sociedades modernas, de tres tipos de convenios. El primero es el asistencial, dominante en los países anglosajones, que basa los derechos en una necesidad perentoria y demostrable que garantice el carácter "merecedor" de quien percibe el beneficio. La práctica de la asistencia social, con su origen en la tradición de la Ley de Pobres de 1834, está caracterizada por la comprobación de los medios de vida y de los ingresos que se poseen. Un segundo tipo de sistema prolonga los derechos con relación al trabajo formal. Esta variante tiene sus orígenes en la tradición de seguros que se desarrolló primero en Alemania y luego en todo el continente europeo. Aquí los derechos están claramente condicionados a una mezcla de vínculos con el mercado laboral y los aportes financieros. El tercer tipo de convenio surge del principio de Beveridge, en el que los derechos se garantizan de manera universal a los ciudadanos, independientemente del grado de necesidad o de su relación con el mercado de trabajo. El ser beneficiario depende, en cambio, de ser ciudadano o de ser residente en el país por un determinado tiempo. Estos sistemas funcionan como tipos ideales, en la realidad todos los países presentan hoy un sistema mixto de estos tres sistemas.

En América Latina, los sistemas de protección social parecen incluir mayoritariamente elementos del segundo tipo de convenio, pero la tendencia desde los años noventa, como uno de los resultados de la profundización de la ofensiva neoliberal, es encaminar el sistema de protección social hacia el modelo asistencialista anglosajón. En ese sentido, Filgueira (1998) plantea que el giro desde un modelo de desarrollo cimentado en el mercado interno hacia uno orientado a la exportación posee su correlato en la transformación del modelo de protección social. De esta forma, sistemas de políticas sociales centralizados, sectorializados y administrados estatalmente van a dar lugar a modelos de políticas sociales descentralizados, focalizados y en algunos casos con delegación en el sector privado, tales como las que se han multiplicado durante las últimas dos décadas.

La proliferación de los PTMC en los países subdesarrollados se dio en medio de un cambio más amplio en la naturaleza del sistema de protección social a nivel mundial. Durante las últimas tres décadas, muchos Estados de Bienestar han pasado a reducir su 
De Prácticas y discursos/ Universidad Nacional del Nordeste/ Centro de Estudios Sociales

tamaño o a ser desmantelados, pasando de sistemas cobertura generalizada hacia sistemas más individualizados, y de prestaciones desmercantilizadoras de bienes y servicios hacia prestaciones que ponen mayor énfasis en las transferencias de efectivo (Lavinas, 2013). Señala la autora que estas diferencias no son para nada triviales, ya que están respaldadas por un gran cambio ideológico con efectos de largo alcance: mientras que una de las funciones del Estado de Bienestar de la posguerra había sido quitar los servicios básicos de salud, educación, vivienda y seguridad social de las manos del mercado -es decir, desmercantilizarlos-, el papel del nuevo modelo busca facilitar el juego de las libres fuerzas del mercado, pasando los servicios públicos a la responsabilidad privada. Mientras que el Estado de Bienestar de la postguerra buscaba reconocer las necesidades y conceder a partir de estas "derechos" garantizando la igualdad de acceso a los bienes públicos, el nuevo modelo ofrece "recompensas" o "premios" a cambio del cumplimiento de obligaciones. Este nuevo esquema de protección social se conoce bajo el neologismo 'workfare' que combina la palabra trabajo (work) con bienestar (welfare).

Este sistema de protección social se basa en una nueva forma de gestionar los riesgos sociales en donde los objetivos de igualdad y justicia social no están ligados a las garantías materiales, sino a las estructuras de oportunidades (Dean, 2007). El centro de las políticas no es distribuir recursos para la población vulnerable, sino mitigar el riesgo y dotar a las personas para que puedan manejar individualmente los riesgos. De acuerdo al Banco Mundial (BM):

"La pobreza tiene relación con la vulnerabilidad, ya que los pobres habitualmente están más expuestos a riesgos, pero al mismo tiempo tienen poco acceso a instrumentos adecuados para el manejo del riesgo. En consecuencia, el suministro y selección de instrumentos adecuados para el manejo social del riesgo se convierte en un medio importante para reducir la vulnerabilidad y proporcionar un medio para salir de la pobreza". (Banco Mundial, 2000:5).

Las viejas formas de gobierno liberal que comenzaron a implementarse luego de los setenta están dando lugar al surgimiento de lo que Rose (1996) llama liberalismo 
De Prácticas y discursos/ Universidad Nacional del Nordeste/ Centro de Estudios Sociales

avanzado, que se caracteriza por promover el autoabastecimiento, el individualismo y la autoresponsabilización. El liberalismo avanzado es crítico de las rigideces de los Estados y de la proliferación de derechos. De este modo, la protección social de los pobres comienza a estar condicionada por su disposición a buscar un empleo, mantenerse capacitado, asistir al sistema de salud o enviar a sus hijos a la escuela. Esto se debe a que los economistas neoliberales asocian pobreza a irresponsabilidades individuales, a su deficiencia para manejar los riegos (Dean, 2007). De este modo, impulsar una mayor gestión y manejo del riesgo "presenta a la Protección Social como una red de protección y a la vez como un trampolín para los pobres" (BM, 2000: 4).

También Barrientos (2004) destaca a nivel latinoamericano el cambio de sistemas estratificados de protección social con beneficios asociados al empleo formal ${ }^{1}$ hacia un sistema en el cual los riesgos afrontados colectivamente se han reducido y han sido reemplazados por estrategias individualizadas. Antes de la emergencia del liberalismo avanzado, los problemas que afectaban a gran parte de la población eran pensados como problemas sociales y tratados, desde la esfera estatal, a partir de un criterio de socialización de los riesgos (Landau, 2008). Con el neoliberalismo, se produce un proceso de reconfiguración de las protecciones sociales, el cual se caracteriza por el pasaje de una cobertura colectiva de los riesgos hacia una cobertura individualizada. Es decir que, con el liberalismo avanzado, se tiende a una individualización del riesgo, en donde cada uno debe convertirse en un gestor de su propia vida, autogobernarse y plantearse como un ser independiente del Estado (Landau, 2008; Procacci, 1999). De este modo, estaríamos en presencia de un período de explosión de los riesgos, de hiperindividualización de las prácticas y de incremento de la mercantilización de las prestaciones; el manejo de los riesgos pasa a ser una estrategia individual y ya no más una empresa colectiva (Castel, 2004).

1 Los trabajadores informales dependían principalmente de sus ingresos y de estrategias familiares para hacer frente a los riesgos. 
De Prácticas y discursos/ Universidad Nacional del Nordeste/ Centro de Estudios Sociales

Hacia 2009, como respuesta a la crisis económica mundial ${ }^{2}$, Naciones Unidas y la OIT plantean el fortalecimiento de la protección social existente y su ampliación por medio de un "Piso de Protección Social", de manera de incorporar a poblaciones vulnerables sin acceso a derechos en materia de protección social (OIT, 2014). Esta iniciativa se dirige a "promover, en el ámbito nacional, estrategias que garanticen un mínimo de acceso a servicios y a la seguridad del ingreso para el conjunto de la población" (OIT, 2012). El organismo plantea que existe una multiplicidad de opciones para cumplir con las garantías básicas, entre ellas, regímenes contributivos, universales, asistenciales, mixtos, políticas activas de mercado de trabajo, programas de garantía de empleo u otros, de conformidad con la Recomendación Nº 202 (OIT, 2014).

De esta forma, en varios países de América Latina se extendió la cobertura mediante PTMC, como un primer paso hacia la propuesta del "piso de protección social", propuesta que busca generar sucesivas ampliaciones en simultáneo; por un lado horizontales, es decir, que apunta a aumentar la cantidad de personas cubiertas y, por el otro verticales, a partir de extender la cantidad de servicios y garantías de seguridad social ofrecidos, así como a mejorar la calidad de las prestaciones. Esta estrategia es presentada en la forma de una "escalera de la seguridad social", que consta de tres escalones con tres distintos niveles de prestaciones, que corresponden a diferentes niveles de garantías. En el centro del modelo se encuentran los regímenes habitualmente contributivos de seguros sociales; entre los afiliados del régimen contributivo obligatorio, los que desean y puedan financieramente buscan una cobertura de nivel cualitativamente más alto -tercer escalón- a través de regímenes complementarios voluntarios -regulados por el Estado-. En el primer escalón se incluiría un gran número de trabajadores, quienes aún no se encuentran cubiertos por los regímenes de seguridad social formales.

Una de las limitaciones de esta perspectiva es que abre una puerta a la discrecionalidad de cada país cuando manifiesta que cada uno de ellos deberá definir los niveles de las

2 La crisis internacional en el 2008 reubica a los sistemas de seguridad social en el centro de la agenda pública, dado que además de atender las cuestiones sociales, actúan como importantes estabilizadores automáticos, cumpliendo un importante rol contracíclico. 
prestaciones monetarias que considere adecuados para una vida digna de acuerdo con las circunstancias nacionales, además de darles libertad para que concreten estas garantías en la forma de prestaciones condicionadas o universales.

Si bien comprendemos como un avance la aplicación de un piso de protección social al conjunto de la población, dado que esto representa un impacto importante en la morigeración de los índices de pobreza y en otorgar ciertos beneficios a los trabajadores informales, entendemos esta propuesta como insuficiente.

Una de las principales dificultades radica en que esta perspectiva no fomenta un cambio significativo dentro de la lógica bismarkiana que ha imperado como paradigma de los sistemas de seguridad social en la mayoría de los países de la región latinoamericana. De este modo, la novedad de esta iniciativa radica en fomentar "una buena coordinación" entre el sistema contributivo y el no contributivo para lograr incluir en alguno de estos dos sistemas al conjunto de la población (OIT, 2012 y 2014). Esto también se observa en la lógica de la "escalera de la seguridad social", en donde se busca cubrir a la totalidad de las personas, pero se ofrecen prestaciones diferenciadas para cada uno de los tres "escalones". Es decir que, el problema, radica en que las prestaciones que garantiza cada uno de estos sistemas se caracterizan por ser desiguales, tanto cuantitativa ${ }^{3}$ como cualitativamente ${ }^{4}$.

Ahora bien, si entendemos que la región latinoamericana se caracteriza por un problema de informalidad laboral de carácter estructural, en donde una importante cantidad de trabajadores de la región no puede acceder a los beneficios de la seguridad social por participar dentro del mercado laboral como trabajadores no registrados, resultaría interesante una propuesta que busque superar la reproducción de la segmentación de los mercados de trabajo en el sistema de protección social.

3 La diferencia en términos cuantitativos radica en que "el diseño del pilar no contributivo debe evitar desincentivar la participación de las personas en el pilar contributivo (mercado de trabajo) por lo cual generalmente entraña beneficios mucho menores que su equivalente contributivo" (OIT, 2012: 17).

4 La diferencia en términos cualitativos radica en la desigualdad de la calidad de las prestaciones y del tipo de garantías que cubre el sistema contributivo y el no contributivo. Además, existen diferencias conceptuales entre un sistema y otro, mientras que las pensiones no contributivas "reemplazan los ingresos de las personas durante la vida activa", las no contributivas tienen como función "garantizar un nivel básico de renta en forma sostenible desde el punto de vista fiscal y justa desde el punto de vista intergeneracional" (CEPAL, 2010: 217 citado por OIT, 2012). 


\section{Capital humano, empleabilidad y distribución del ingreso: ¿El fin de la pobreza en el corto y largo plazo?}

\subsection{El diagnóstico}

Desde algunas instituciones -especialmente desde el Banco Interamericano de Desarrollo-BID- se sostiene que los PTMC son un producto endógeno de América Latina (Stampini y Tornarolli, 2012); sin embargo, algunos autores demuestran que sus antecedentes intelectuales se encuentran más al norte (Lavinas, 2013), ya que conceptualmente se basan en la confluencia de dos ideas: aquella que refiere a la teoría de capital humano, con la que se pretende erradicar la pobreza en el largo plazo, por un lado; y aquella que se basa en luchar contra la pobreza en el corto plazo a partir del gasto social orientado,-o focalizado-, por el otro. Se entiende que los PTMC parten del principio de focalización, pero también tienen un carácter filantrópico ya que las contraprestaciones que realizan los beneficiarios serán provechosas para ellos en el largo plazo (Lavinas, 2013). Esta idea tiene su pilar central en la teoría del capital humano de los economistas de la Escuela de Chicago, puntualmente en Schultz y Becker, en donde el capital humano se ve como un elemento importante para el éxito del desarrollo de un país ${ }^{5}$. Esta teoría correlaciona los años de escolaridad con los ingresos futuros, en donde una mayor inversión en capital humano significará más adelante un empleo de salario superior ${ }^{6}$.

La estrategia para cortar con la transferencia intergeneracional de la pobreza se sustenta en la idea de que el aumento del capital humano derivará en un incremento

\footnotetext{
5 Para una mejor aproximación a la Teoría de Capital Humano, consultar Schultz (1961) y Becker (1983), entre otros. Para un abordaje que enfatiza otras perspectivas teóricas que discuten con la Teoría de Capital Humano, consulte Albano y Salas (2007) o Morduchowicz (2004).

6 Esta idea se encuentra tan latente en la ejecución de este tipo de programa que se han desarrollado algunos ejercicios matemáticos para calcular su impacto futuro. Skoufias (2000) señala que a partir de estimaciones sobre el Programa Progresa de México se calculó que como resultado del incremento 0.66 años de escolaridad, los salarios de los futuros trabajadores podrían ser $8 \%$ mayores y que, como resultado de los complementos alimenticios, los niños en el futuro podrían obtener un incremento de $2.9 \%$ en sus ingresos.
} 
De Prácticas y discursos/ Universidad Nacional del Nordeste/ Centro de Estudios Sociales

de la empleabilidad que permitirá a los beneficiarios salir de su condición de pobres, ya que podrán incorporarse, en un futuro, al mundo laboral, o podrán conseguir empleos de mayores ingresos. Los niveles educativos generalmente bajos y la salud vulnerable de los pobres son vistos como un obstáculo para la prosperidad debido a que les impiden participar plenamente en el mercado.

La empleabilidad ${ }^{7}$ es entendida como la probabilidad que tiene una persona de obtener un empleo, y en el caso de los programas bajo análisis, se estaría promoviendo un tipo de empleabilidad que Gazier (1990a) denomina iniciativa y combina actitudes, habilidades y calificaciones consideradas indispensables para enfrentar los rápidos cambios en una economía globalizada. También apunta a la responsabilidad individual y a la capacidad de construir y movilizar redes sociales. Cada trabajador aparece en esta visión como gestor de su propia trayectoria laboral, remarcando el carácter individual que determina el acceso o no al mundo del trabajo y, por tanto, el destino de ser o no ser pobre. Formación y activación persiguen un mismo objetivo: aumentar la autonomía y las oportunidades de los individuos -lo que en la literatura anglosajona se conoce como empowerment- para darles los medios para llevar adelante sus proyectos en lugar de simplemente asistirlos. El rol del Estado es ahora proveer a los individuos de una parte de estos activos o ayudarlos a adquirirlos -lo que Gautié (2004) denomina Estado Social Patrimonial-, de manera que ellos puedan disponer de un patrimonio que los constituya como "empresarios" de su propia vida.

Con el proceso de activación, se estaría explorando una nueva zona entre la indemnización y la garantía del empleo: el de la inserción, en donde los beneficiarios tendrían obligaciones positivas y serían considerados ciudadanos activos (Rosanvallon, 2007). Dubet (2010) introduce el concepto de sociedad activa para dar cuenta de las sociedades que promueven el proceso de activación, en las que los ciudadanos merecen ayuda y oportunidades siempre y cuando acepten movilizarse, actuar y tener sus propios proyectos para salir adelante. Dentro de estas sociedades, la solidaridad se daría a cambio de la actividad.

7 Para una discusión de diferentes perspectivas del concepto, ver Barbier, 2000; Gazier, 1990a y 1990b. 
De Prácticas y discursos/ Universidad Nacional del Nordeste/ Centro de Estudios Sociales

Sin embargo, a partir de esta concepción, se estaría excluyendo la posibilidad del reconocimiento de los problemas de la pobreza y el desempleo como consecuencia de cuestiones más estructurales ligadas al modo de acumulación adoptado, a las características estructurales que presentan los mercados de trabajo latinoamericanos, a las políticas macroeconómicas desarrolladas, etc.; esta visión de la problemática social parece algo sesgada y tiende a culpar a la víctima del problema que padece sacando el foco de los problemas estructurales que presentan los países subdesarrollados pobres para poner el eje de la pobreza y la exclusión en la falta de capital humano, cuyo incremento, desde esta teoría, resolvería tanto los problemas del desempleo como de la pobreza. Desde nuestra perspectiva, el incremento del desempleo y del subempleo, así como el aumento de la precariedad laboral, la pobreza y la indigencia, son consecuencias del propio funcionamiento del sistema económico y de las características particulares que adopta la región latinoamericana en este contexto y requieren de políticas estructurales para combatirlos.

\subsection{El consenso}

Al entender que prestar atención a la problemática social con los sistemas clásicos en América Latina compromete gran parte de los recursos públicos, los organismos internacionales recomiendan focalizarse en los más pobres, otorgarles herramientas activos- para mejorar su gestión de los riesgos -a partir de la exigencia de contraprestaciones- y, además, incentivar su inserción económica. Es en este sentido que la perspectiva de los PTMC es considerada como la que mejor utiliza los fondos públicos direccionándolos hacia aquellas personas con mayores necesidades.

La focalización del gasto se materializa en programas de amplia cobertura y de muy bajo costo. Un estudio de la CEPAL (2011a) señala el importante crecimiento que experimentó este tipo de programas en la región latinoamericana durante el último decenio: mientras que la cobertura (\% del total de la población) pasó de 5,7\% en el año 2000 a un 19,3\% en el año 2010, el presupuesto ejecutado (\% de PBI) creció de 0,2\% a 
De Prácticas y discursos/ Universidad Nacional del Nordeste/ Centro de Estudios Sociales

$0,4 \%$. Este es uno de los principales atractivos para los gobiernos ya que pudieron ampliar su política social sin realizar un gran esfuerzo fiscal.

A continuación se presenta un gráfico en el que se puede apreciar la variación entre los distintos países de la región en relación con estos dos indicadores.

\section{Gráfico 1}

Cobertura (en \% sobre la población total) y Presupuesto Ejecutado (\% de PIB) para América Latina y El Caribe, 2010. 
De Prácticas y discursos/ Universidad Nacional del Nordeste/ Centro de Estudios Sociales

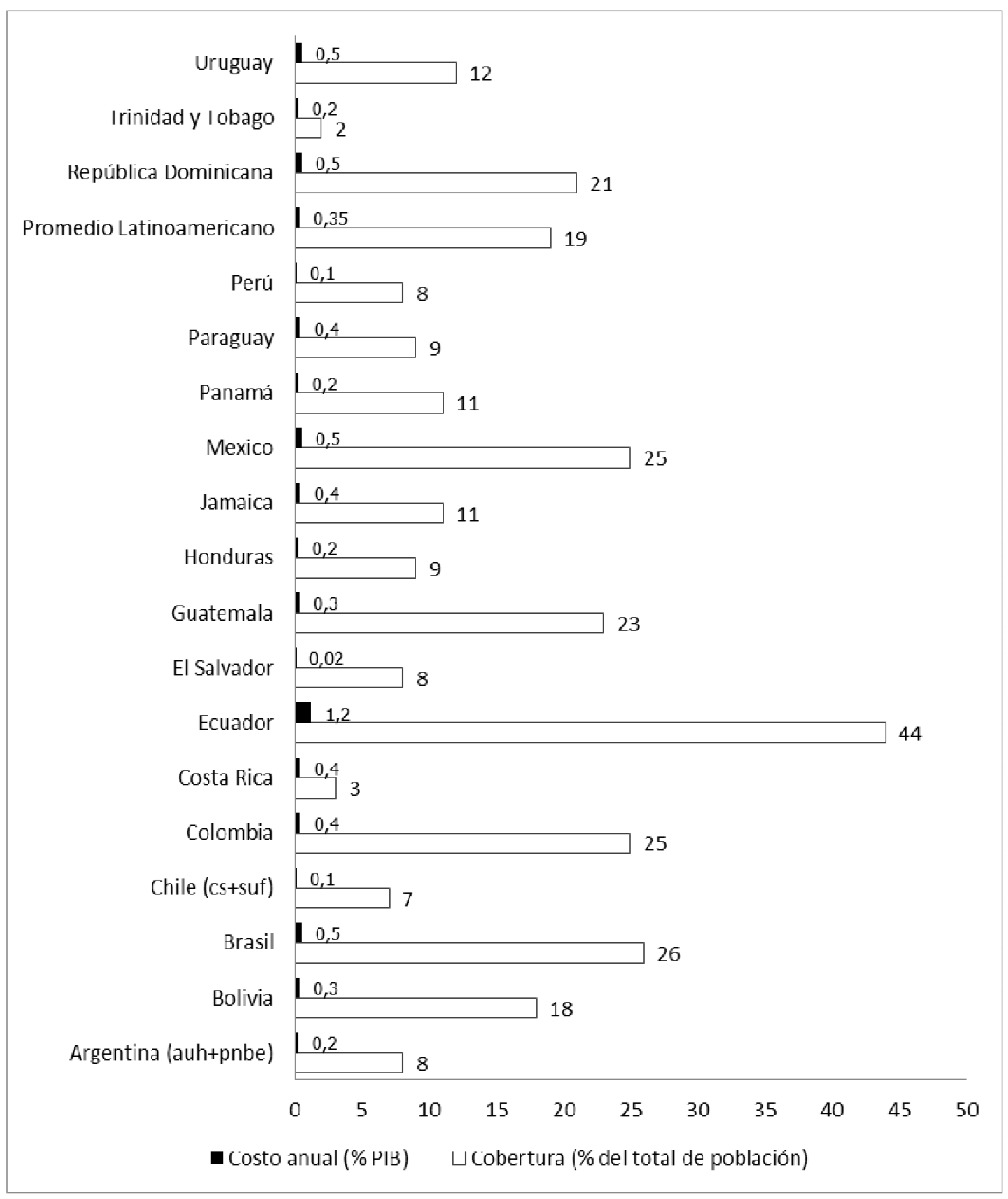

Fuente: Elaboración propia en base a datos de la CEPAL ( 2011b)

Una de las principales razones por la que la cobertura es amplia, pero el presupuesto acotado, es el interés porque todos los programas tengan un potencial 
De Prácticas y discursos/ Universidad Nacional del Nordeste/ Centro de Estudios Sociales

desmercantilizador ${ }^{8}$ acotado, lo que se materializa en programas de beneficios bajos. Por este motivo, estos PTMC buscan que la posesión de un empleo garantice un ingreso mayor que una prestación social, preservando el atractivo de las remuneraciones recibidas en el mercado de trabajo.

De hecho, el Banco Mundial (2000) señala que hay que ser muy cautelosos al pactar el valor de las prestaciones dinerarias entregadas a los beneficiarios, ya que estas pueden tener efectos negativos sobre el desarrollo económico de un país, principalmente porque pueden reducir los esfuerzos individuales, por ejemplo, en la búsqueda o aceptación de un trabajo; esto mismo plantea la OIT (2012) al señalar que el diseño del pilar no contributivo -en donde incluyen a los PTMC- debe evitar desincentivar la participación de las personas en el pilar contributivo. Sin embargo, la baja cuantía de los beneficios termina impactando sobre el potencial del programa para reducir la pobreza: el efecto de los programas sobre la pobreza en el corto plazo es moderado, dado el peso de la transferencia monetaria en comparación con el consumo familiar o con la línea de pobreza, que suelen estar a nivel latinoamericano en alrededor del 20$21 \%$ y del $70-90 \%$, respectivamente (Valencia Lomelí, 2008). Entonces este tipo de programas puede ser exitoso en reducir la brecha o la severidad de la pobreza, pero puede tener un muy pequeño impacto sobre la tasa de pobreza (Skoufias, 2000).

\section{Los Programas de Transferencias Monetarias Condicionadas (PTMC) en América Latina}

Líneas arriba hemos señalado que los PTMC comienzan a implementarse en América Latina durante la década del 90. Hacia fines de esta década se ejecutaban a nivel regional solamente algunos programas de estas características. Sin embargo, su proliferación durante los 2000 fue significativa, ocho años más tarde todos los países

\footnotetext{
8 A diferencia del concepto de desmercatilización utilizado previamente, en este caso hacemos referencia a aquel desarrollado por Esping-Andersen (1993), basándose en las tesis de Polanyi (2004) y, posteriormente, de Offe (1990). Este concepto hace referencia al grado en el que los individuos o las familias pueden mantener un nivel de vida socialmente aceptable, independientemente de su participación en el mercado laboral a través de la venta de su fuerza de trabajo.
} 
De Prácticas y discursos/ Universidad Nacional del Nordeste/ Centro de Estudios Sociales

de la región latinoamericana implementaban al menos uno de estos programas y comenzaban a expandirse hacia otras regiones -tales como India, Turquía, etc.llegando a implementarse PTMC en más de 30 países del mundo.

Lena Lavinas (2013) determina tres razones de esta rápida expansión en América Latina. En primer lugar, la elección de una ola de gobiernos progresistas que empujó las preocupaciones sociales a la agenda política en Latinoamérica. En segundo lugar, la autora destaca que el continente a partir de los 2000 experimenta un periodo renovado -aunque desigual- de crecimiento económico, en donde los buenos precios internacionales de los commodities impulsaron ingresos por exportaciones, y que también la crisis subprime de 2008 generó el ingreso masivo de dinero en busca de más rentabilidad en los mercados emergentes. Esto dio a los gobiernos un margen de maniobra fiscal del que habían carecido anteriormente. Finalmente, un tercer factor crítico fue institucional, ya que luego de su escepticismo inicial, el Banco Mundial y otros organismos internacionales de crédito buscaron promover los PTMC como parte de su nueva estrategia de gestión social del riesgo sobre la que hicimos referencia líneas arriba.

La estructura básica de los PTMC consiste en la entrega de recursos monetarios y no monetarios a familias o personas en situación de vulnerabilidad -ya sea de ingresos o de vulnerabilidad laboral- con la condición de que los perceptores cumplan con ciertas conductas asociadas al aumento de capital humano y a la mejora de la empleabilidad para que puedan luego ingresar a algún trabajo lucrativo. Otro aspecto común es la focalización de estos programas, que se basa en la adopción de criterios técnicos a partir de los cuales se selecciona a los destinatarios. La selección generalmente se basa en procedimientos en dos o más etapas, con predominio de la focalización por unidades geográficas y los métodos de selección de personas u hogares por comprobación indirecta de los medios de vida/ingresos (CEPAL, 2011a).

Sin embargo, pese a la existencia de estos rasgos comunes, un estudio pormenorizado de los distintos PTMC implementados en la región muestra que esta reconfiguración de las políticas sociales no ha sido homogénea. Proponemos entonces diferenciar a los PTMC en tres tipos, teniendo en cuenta la problemática a la que buscan dar respuesta y 
De Prácticas y discursos/ Universidad Nacional del Nordeste/ Centro de Estudios Sociales

las características de la población a la que se dirigen. Un primer tipo incluye a aquellos programas que tienen como objetivo cortar con la transferencia intergeneracional de la pobreza y generalmente están destinados a familias en situación de vulnerabilidad, especialmente aquellas con presencia de niños. Un segundo tipo es aquel que busca promover la inserción laboral de las personas en edad de trabajar a partir de la activación de la protección social. Y, finalmente, el tercer y último tipo tiene como objetivo otorgar beneficios a trabajadores informales, que por las características propias de su inserción laboral no tienen acceso a los derechos provistos por la seguridad social.

\subsection{Pobreza y PTMC}

La mayoría de los PTMC son programas que transfieren ingresos mensualmente a las familias en situación de pobreza o indigencia condicionados al cumplimiento de determinados deberes que se vinculan, en la mayoría de los casos, con la realización de controles sanitarios, nutricionales y la asistencia a centros educativos.

Estos programas se crearon con la intención de modificar las estrategias familiares utilizadas para hacer frente a una diversidad de riesgos sociales, tales como las crisis económicas, las catástrofes familiares o naturales. Esto se debe, por un lado, al reconocimiento de que en los hogares más pobres suele hacerse frente a la disminución de los ingresos reales con la incorporación de mujeres, jóvenes y niños al mercado laboral a partir de diversas formas de empleo precario; $y$, por el otro, a que esto genera impactos negativos de largo plazo, especialmente en lo que respecta a la acumulación de capital humano, ya que en muchos casos se reemplaza escolaridad por trabajo (Barba Solano y Valencia Lomelí, 2011). El Banco Mundial, en su informe sobre la nueva gestión social del riesgo del 2000 (BM, 2000: 18), plantea que muchas veces ante catástrofes sociales o naturales "las estrategias de la superación de la pobreza suelen basarse en mecanismos informales tales como el desahorro/endeudamiento individual, la migración, la venta de la mano de obra (incluyendo la de los niños), la 
De Prácticas y discursos/ Universidad Nacional del Nordeste/ Centro de Estudios Sociales

reducción de la ingesta de alimentos", descuidando así la inversión en capital humano y perpetuando "la pobreza".

Por este motivo, un tipo característico de PTMC aplicado en la región es aquel que tiene como población objetivo a los niños de las familias en situación de vulnerabilidad. Estos programas se desarrollan con el fin de incrementar el capital humano de estos con educación y salud- para así cortar con la transferencia intergeneracional de la pobreza (Barrientos, 2011) articulando objetivos de corto y largo plazo.

En algunos casos, a estos objetivos se les agrega uno de mediano plazo, que se caracteriza por exigirles a los adultos, por ejemplo, la participación en talleres y sesiones de aprendizaje de habilidades para la vida; es decir, la participación en programas de desarrollo de capacidades para los adultos y en programas de activación (CEPAL, 2007) como los que serán desarrollados en la próxima tipología.

En líneas generales, se pueden evidenciar impactos positivos en lo que respecta a la disminución de los niveles de indigencia como resultado de la aplicación de estos programas, y de la pobreza en el corto plazo; sin embargo, en algunos casos pese a la amplia cobertura que presenta este tipo de programas, esta no pareciera ser suficiente para los niveles de pobreza que experimenta la región (CEPAL, 2007). Además, cabe aclarar que este impacto es significativo especialmente cuando la medición de pobreza se hace por ingresos ${ }^{9}$, pero pierde relevancia cuando se quiere observar su impacto sobre la pobreza estructural, generalmente asociada a la satisfacción de necesidades básicas vinculadas al acceso y las condiciones habitacionales -vivienda-, servicios sanitarios, educaciones, etc., de los hogares, cuestiones cuya resolución implicaría otro nivel de compromiso de las políticas públicas.

De hecho, si bien puede considerarse que estos programas son valiosos en varios sentidos -disminuyen la pobreza extrema, promueven la asistencia de los niños a la escuela, aumentan su acumulación de años promedio de escolaridad, disminuyen la

9 Esta metodología es conocida como el método indirecto de medición de la pobreza y se caracteriza por contrastar los ingresos de un hogar con una canasta básica de alimentos normalizada. 
De Prácticas y discursos/ Universidad Nacional del Nordeste/ Centro de Estudios Sociales

desnutrición infantil, diversifican la alimentación familiar, impulsan la asistencia de los niños a centros de salud, así como logran disminuir las tasas de morbilidad infantil, maternal y juvenil-, no se han corroborado otros resultados esperados como la ruptura en la reproducción intertemporal de la pobreza (Barba Solano y Valencia Lomelí, 2011). Esto se debe, por una lado, a que no se ha constatado que un aumento de la escolarización se vea reflejado en un aumento del capital humano; y, por el otro, no hay evidencia que sustente que la transferencia de ingresos a sectores vulnerables se materialice en una disminución del trabajo infantil o juvenil (Barba Solano y Valencia Lomelí, 2011). Además, en muchos casos, el impacto de los PTMC también está circunscripto a la calidad y posibilidad de acceso a los servicios públicos prestados, ya sea en materia de educación como de salud. De hecho, se critica que estos programas han generado un incremento en la demanda de servicios públicos, pero no se realizaron las inversiones necesarias para una posible ampliación en la oferta de estos servicios o para mejorar su calidad, cosa que probablemente requeriría una ejecución presupuestaria de mayor envergadura.

\subsection{Los PTMC y la activación de la protección social}

Otro conjunto de PTMC intenta dar respuesta a los problemas del desempleo y de la exclusión creciente mediante hacer que el trabajo pague. Estos programas proponen vincular más estrechamente las prestaciones de mantenimiento de ingresos -como el seguro de desempleo- con medidas activas de formación e incentivos a la rápida inserción laboral. Por ello es conocido como el paradigma de activación de las políticas sociales y de empleo.

A estos PTMC se los puede agrupar en dos subconjuntos; por un lado, aquellos programas que buscan incrementar el capital humano y generan un círculo de habilidades y empoderamiento de los desocupados -a través de sistemas de educación, capacitación y formación profesional- y, por el otro, aquellos que buscan incentivar el abandono por parte de los beneficiarios de las políticas de asistencia social en miras de que se inserten rápidamente al mercado de trabajo -agencias de colocación de 
De Prácticas y discursos/ Universidad Nacional del Nordeste/ Centro de Estudios Sociales

desocupados, incentivo a microemprendimientos, reducciones de los beneficios, sanciones, etc.-. En diversas ocasiones podemos encontrar programas que vinculan estos subconjuntos, por ejemplo, aquellos que buscan aumentar el capital humano y al mismo tiempo plantean un sistema de alícuotas decrecientes.

Si bien existen diferencias al interior de los programas de activación, es posible destacar ciertas similitudes, como es el énfasis que todos estos ponen en incrementar la autonomía y las oportunidades de los individuos para que estos puedan, por sus propios medios, lograr sus proyectos.

La principal característica de estos programas activos es que vinculan el otorgamiento de ingresos a grupos vulnerables con la obligatoriedad de realizar contraprestaciones que les permitan insertarse nuevamente en el mundo laboral. Las contraprestaciones, en su gran mayoría, se vinculan con la participación en talleres de inserción ocupacional, programas de capacitación, prácticas calificantes, entrenamiento en los puestos de trabajo o terminalidad educativa. También pueden encontrarse contraprestaciones que impliquen la postulación a determinados puestos de trabajo o a aceptar la oferta laboral propuesta desde las oficinas de empleo. Esta es una de las maneras en la que estos PTMC buscan que los desocupados mantengan ciertos hábitos vinculados al mercado laboral ya que, desde diversos espacios académicos y políticos, se postula que el otorgar un ingreso sin contraprestación desincentivaría la cultura del trabajo de manera que quienes cobren ese beneficio perderían el interés en buscar un empleo. Además, de este modo se conseguiría mayor consenso en su implementación desde la opinión pública debido a que no se estaría dando dinero a cambio de nada (Lavinas, 2013) y así se disiparían los miedos acerca de los efectos de cronificación que podría llegar a generar la política social sin contraprestación (Pérez Eransus, 2006). De hecho, algunos estudios muestran que la existencia de contraprestaciones no generó un impacto importante en las reinserciones laborales, sino que solo contribuyeron a aumentar los costos administrativos y burocráticos en su implementación (Lavinas, 2013).

Estas contraprestaciones han sido objeto de diversas críticas, entre ellas podemos señalar aquellas que plantean que la exigencia a tomar un trabajo por parte de los 
perceptores de los programas muchas veces los obliga a tomar cualquier tipo de empleo sin importar la calidad del mismo, generando inserciones laborales precarias. En este mismo sentido, Pérez Eransus (2006) se pregunta: ¿Hasta qué punto el acceso a cualquier tipo de empleo no termina contribuyendo a la extensión de la precariedad laboral?

Por otro lado, este tipo de PTMC retoma la idea de generar un potencial desmercantilizador moderado -nuevamente en los términos de Esping-Andersen (1993) antes planteados-. Una de las estrategias utilizadas para esto suele ser otorgar beneficios decrecientes en los que la cuantía percibida por los beneficiarios vaya disminuyendo a medida que pasa el tiempo, como una forma de desincentivar la permanencia en el programa.

Sin embargo, este último razonamiento también ha sido fuertemente criticado. Se cuestiona su arraigo a las corrientes de pensamiento ortodoxas, en las que se fundamenta que el desempleo es el resultado de una elección racional ${ }^{10}$ que la persona hace entre la dicotomía trabajo/ocio. Según esta teoría, si no se incentiva a los trabajadores a reinsertarse en el mercado de trabajo, los desocupados caerían en la trampa de la pobreza ${ }^{11}$. Lejos de compartir los postulados que esta teoría promueve, entendemos que son diversos los factores por los que una persona puede permanecer en condición de desempleo ${ }^{12}$, y que estos factores van a entenderse en mayor media como consecuencias de las características que adopta el mercado de trabajo en épocas contemporáneas -signado por el desempleo, la inestabilidad y precariedad laboral-.

10Esta teoría sostiene que la conducta del desempleado o inactivo se define como el resultado de un cálculo racional eligiendo entre ocio/trabajo- en el que la propensión al ocio resulta favorable y, como consecuencia, se opta de forma voluntaria por la inactividad.

11La trampa de la pobreza expresa que, ante la posibilidad de cobrar un subsidio de forma indefinida, trabajar no tendría sentido porque el incremento en los ingresos netos es pequeño o nulo, o porque los inconvenientes que acarrea un empleo son grandes. Acceder a un empleo solo sería racionalmente aceptable, según esa teoría, cuando la diferencia de ingresos entre la actividad y la inactividad es amplia, lo que ocurre cuando los salarios son altos y/o las prestaciones bajas.

12La presencia de cargas familiares no compartidas, la presencia de problemáticas relacionadas con la salud no reconocidas como incapacitantes, la discriminación laboral de grupos vulnerables como jóvenes, mujeres y mayores de 45 años o la ausencia de oportunidades de trabajo (Pérez Eransus, 2006). 
De Prácticas y discursos/ Universidad Nacional del Nordeste/ Centro de Estudios Sociales

En esta misma línea se ha cuestionado el énfasis que pone este paradigma de activación sobre la inserción laboral argumentando que esto representa un riesgo de reduccionismo sobre los problemas reales que afectan a las personas que demandan este tipo de prestaciones económicas, en donde la carencia de un empleo no es el único factor problemático, sino que muchas veces existen también carencias de vivienda, salud, educación, etc. Pérez Eransus (2006) señala que diversas investigaciones han demostrado correlación entre el éxito de los dispositivos -la inserción en el mercado- y las condiciones iniciales de los desocupados/excluidos. Es decir que aquellas personas que cuenten con un mayor nivel de deterioro personal y social tendrán mayores dificultades, o directamente no podrán cumplir con los requisitos establecidos en las contraprestaciones de activación implicando un riesgo creciente a la desprotección social de los sujetos más débiles.

El proceso de activación estaría basado en promesas tácitas hacia los beneficiarios: 1 . la promesa de que la pobreza va a disminuir si las personas comienzan a aceptar los trabajos propuestos; 2 . en relación con los beneficiarios que transitan por programas de capacitación y aumento de la empleabilidad, la promesa tácita de que capacitándose tendrán cada vez más oportunidades de empleo y de empleos de mayor calidad; 3. la promesa de que aumentará la inclusión de las personas si estas aceptan las tareas propuestas (Barbier y Knuth, 2010).

Se podría hacer una última reflexión respecto a la idea propuesta desde este tipo de PTMC y desde los organismos internacionales de crédito, de considerar el acceso al empleo como una valiosa herramienta de integración social. El Banco Mundial sostiene que en el objetivo por garantizar la inclusión social hay que darle la posibilidad a los pobres de "al menos, retomar un trabajo lucrativo" (BM, 2000: 3). Sin embargo, coincidimos con Pérez Eransus (2006) en que pensar la inclusión social a partir del empleo genera plantearse ciertos interrogantes: ¿Cualquier forma de empleo mercantil es integradora?, ¿cuáles son los efectos negativos que la precariedad en el empleo puede ejercer en las personas que viven en situaciones de pobreza? En un contexto de profundización de las problemáticas ligadas al empleo, es preciso repensar las estrategias de activación con los más vulnerables, ya que las condiciones de 
De Prácticas y discursos/ Universidad Nacional del Nordeste/ Centro de Estudios Sociales

explotación de algunos empleos, lejos de favorecer la integración, estarían empeorando la situación de exclusión de algunos hogares (Pérez Eransus, 2006).

\subsection{Informalidad laboral y PTMC}

Finalmente, podemos señalar un último tipo de PTMC, cuya implementación se justifica principalmente con el objetivo de dar respuesta a los altos niveles de informalidad y precariedad laboral que caracterizan a los mercados de trabajo latinoamericanos. Esto permitiría correr el eje que fundamenta la aplicación de los PTMC ya que, en este caso, y a diferencia de los anteriores, se toma como referencia a la situación ocupacional de las personas activas para darles coberturas a aquellos que se encuentran trabajando, pero que no están registrados ni aportando al sistema de seguridad social, y, por lo tanto, no se encuentran protegidos por este (Lo Vuolo, 2009). Sin embargo, continúan estando condicionados por ingresos, es decir, son programas que tienen como población objetivo a trabajadores informales de bajos ingresos.

La región latinoamericana presenta un sistema de asistencia social truncado debido a que las altas tasas de informalidad y precariedad dejan sin cobertura a un gran número de trabajadores que queda excluido del régimen de la seguridad social (Barrientos, 2011). En el 2013, el 47\% de los trabajadores de América Latina y el Caribe se desarrolla dentro de la economía informal, ya sea por tener un empleo precario o por realizar sus actividades dentro del sector informal (OIT, 2013). Es decir que casi la mitad de los trabajadores de la región no se encuentra protegida por el sistema de seguridad social. Esta situación se agrava si se tiene en cuenta que en muchos casos estos empleos informales se encuentran signados por distintas vulnerabilidades asociadas al empleo irregular, a períodos de bajos ingresos y al desempleo (Barrientos, 2011). De allí que con la expansión de la protección social a partir de la implementación de PTMC y la expansión de pensiones no contributivas, se busca reducir las brechas entre los trabajadores no beneficiados por el sistema contributivo y aquellos que tienen acceso a empleos registrados en el sistema de seguridad social, buscando también recomponer ingresos y aliviar la condición de los trabajadores pobres. 
De Prácticas y discursos/ Universidad Nacional del Nordeste/ Centro de Estudios Sociales

De hecho, algunos autores señalan que la focalización -en los trabajadores informales pobres- ha sido pensada como una estrategia para contrarrestar la desigualdad que genera un sistema de seguridad social basado en el convenio corporativo. El argumento central respecto a la focalización es que el modelo de política social instaurado a partir de la segunda posguerra no había tenido la capacidad de atender a los sectores más pobres y había sido aprovechado básicamente por los sectores obreros organizados sindicalmente y por las clases medias urbanas (Isuani, 2012). Basándose fundamentalmente en el desempeño de la seguridad social, se reconocía que los sectores de menores ingresos no poseían ni cobertura previsional, ni protección contra la enfermedad o el desempleo. En consecuencia, para lograr mayor equidad social y llegar efectivamente a los más pobres, debía remodelarse el edificio de la política social: la focalización del gasto público en dichos sectores sería la herramienta que produciría esta transformación.

Uno de los principales avances que se pueden establecer con la implementación de estos programas se vincula con el reconocimiento de la precariedad y la informalidad como una problemática a atender por los distintos gobiernos. Como se señaló párrafos arriba, como consecuencia de las características estructurales de los mercados de trabajo latinoamericanos, quedaría una gran cantidad de trabajadores excluidos del sistema contributivo de la seguridad social y, por tanto, un gran porcentaje de personas desprotegidas frente a los diversos riesgos que aquejan a nuestras sociedades.

Paradójicamente, si bien se trata de reconocer los derechos laborales para aquellos trabajadores en situación de vulnerabilidad laboral, dentro de las críticas principales que pueden hacerse a estos programas podemos destacar, por un lado, que sus beneficiarios no son reconocidos como sujeto de derecho, sino como beneficiarios de programas sociales $y$, por el otro, que la estabilidad de estos programas en el largo plazo es incierta, por lo que tampoco se puede afirmar que estos programas reconozcan derechos en el largo plazo. Si bien difícilmente algún gobierno esté dispuesto a afrontar el costo político que implicaría dar de baja algunos de estos programas, de amplio consenso a nivel latinoamericano, congelar los ingresos 
De Prácticas y discursos/ Universidad Nacional del Nordeste/ Centro de Estudios Sociales

transferidos a los beneficiarios en un período inflacionario bastaría para desarticularlos, ya que no hay ninguna ley que respalde la indexación del beneficio.

Dado que uno de los objetivos de este tipo de programas es la reducción de las desigualdades entre trabajadores registrados y aquellos que no lo están, no se entiende por qué se exige realizar contraprestaciones a los beneficiarios del programa, pero no a aquellos que pertenecen al sistema contributivo. Nuevamente la hipótesis que plantean sus defensores es que los trabajadores en empleos precarios se encuentran en un mayor nivel de vulnerabilidad social y económica y, por tanto, la exigencia de contraprestaciones que aumenten el capital humano de los niños que se encuentran dentro de esos núcleos familiares podrían fortalecer las posibilidades futuras de estos para insertarse en empleos de mayor calidad y estabilidad. Otra hipótesis es que el exigir una contraprestación a cambio de los beneficios se sustentaría, una vez más, en la "ética del trabajo", generando así mayor empatía en las clases medias y una mayor aceptación de la sociedad.

Finalmente, con este tipo de PTMC se refuerza la idea de vincular el otorgamiento de subsidios al fomento de la relación beneficiario-mundo laboral, lo cual encontraría una mayor aceptación desde los distintos actores de la sociedad en toda la región (Bonvecchi y Smulovitz, 2008). Aquellas políticas que se desvinculan del mundo laboral para el otorgamiento de ingresos plantean la complejidad de romper con un paradigma que lleva cientos de años vigente, en el que la inserción al mundo laboral es entendido como mecanismo fundamental para garantizar un ingreso $y$, por ende, el acceso a la satisfacción de las necesidades humanas (Isuani, 2008). Es por este motivo que no sería fácil justificar la noción de otorgar un ingreso sin que el trabajo sea su contracara. Según este autor, la mirada culturalmente prevaleciente plantea que el trabajo sigue siendo la principal vía de inserción social y que, por tanto, las políticas de ingresos se justifican siempre que haya una labor de contraparte.

\section{Reflexiones finales}


De Prácticas y discursos/ Universidad Nacional del Nordeste/ Centro de Estudios Sociales

En el presente artículo hemos analizado algunas de las características que adopta en América Latina la reconfiguración del sistema de protección social. Señalamos como los PTMC se enmarcan en esta reformulación de la política social a nivel regional, en la que se adhiere al nuevo esquema de protección social conocido como workfare. A partir de un estudio de los distintos PTMC implementados en Latinoamérica, hemos determinado cuál es el diagnóstico general en el que estos se sustentan y cuáles son las causas del consenso generalizado de su implementación. También nos hemos preguntado si esta reconfiguración de las políticas sociales se ha dado de manera homogénea o si existen particularidades al interior de los PTMC, cuyo estudio nos permitió distinguir tres tipos de PTMC que se diferencian por el grupo objetivo al que se dirigen y por el problema social sobre el que se focalizan -pobreza, desempleo o informalidad-.

Una primera reflexión es que si bien los PMTC son valiosos en varios sentidos disminuyen la pobreza extrema, promueven la asistencia de los niños a la escuela, aumentan su acumulación de años promedio de escolaridad,entre otros-, no se han corroborado otros resultados esperados como la ruptura en la reproducción intergeneracional de la pobreza o la seguridad alimentaria de la población. El impacto sobre la pobreza en el corto plazo ha sido importante, pero principalmente cuando su medición es por ingresos; el impacto sobre la pobreza estructural ha sido moderado.

Los PTMC permiten que sectores de población antes excluidos de las posibilidades de consumo puedan acceder a bienes de primera necesidad, pero no garantizan ni la salida de la pobreza, ni mayores posibilidades de movilidad ascendente. De hecho, detrás de estos programas podemos encontrar el concepto de equidad -que busca igualar las oportunidades individuales al otorgar a los sujetos beneficios socialmente considerados como básicos-, pero no promueve un reconocimiento del otro como un igual, ni una disminución de las desigualdades dentro de la sociedad. A su vez, dichos programas no generan la titularidad de derechos y fomentan mecanismos de control y gestión de la pobreza por parte del Estado. Por su parte, si bien se promueve el acceso de las personas pobres a servicios de primera necesidad vinculados a salud y educación, no se ha trabajado lo suficiente en ampliar la oferta y la calidad de estos 
De Prácticas y discursos/ Universidad Nacional del Nordeste/ Centro de Estudios Sociales

servicios, ni en generar mayor homogeneidad de prestaciones con el resto de la sociedad.

Hemos destacado a lo largo de este documento que estos programas se caracterizan por presentar una amplia cobertura a un bajo costo, de ahí gran parte de su popularidad y evidente consenso, pese a lo cual se evidencian dificultades cuando se evalúa la sustentabilidad del financiamiento a largo plazo, especialmente para los países de menores ingresos, los cuales apelan a créditos otorgados por organismos internacionales para tal finalidad. De hecho, en la mayoría de los casos, los PTMC no se sustentan en un avance material de reformas impositivas y de mecanismos de redistribución más igualitaria de la riqueza, sino en la utilización de recursos públicos ya existentes, a veces del sistema contributivo, o la demanda de créditos internacionales. Una fiscalidad regresiva -como la predominante en América Latina- vuelve difícil un aumento de las transferencias sociales a la altura de lo que está en juego (Salama, 2011).

Cabe preguntarse entonces por posibles alternativas a los problemas de exclusión y desempleo vigentes. Se ha avanzado en el debate entre académicos y organizaciones sociales sobre la posibilidad de avanzar hacia otras formas de inclusión que vayan más allá del mundo laboral. En las últimas décadas, el fenómeno de los trabajadores pobres comenzó a interpelar al empleo y a los ingresos que este genera como único mecanismo de inclusión y salida de la pobreza.

El enfoque de derechos interpreta a la asistencia y a la protección social como mecanismos orientados a garantizar el ejercicio igualitario de los derechos económicos, sociales y culturales (DESC) del conjunto de la población. De este modo, las políticas sociales se convierten en la cristalización de las obligaciones del Estado frente a la ciudadanía, exigibles en virtud del cumplimiento de las garantías constitucionales y los tratados y pactos internacionales sobre derechos humanos (Abramovich, 2006; Abramovich y Pautassi, 2006; Pautassi, 2010). Otras perspectivas promueven la implementación de un ingreso ciudadano a la niñez como primer paso hacia un ingreso ciudadano pleno para toda la población. Se busca romper con algunos de los principales problemas que presentan los PTMC a partir de promover la universalidad, la 
De Prácticas y discursos/ Universidad Nacional del Nordeste/ Centro de Estudios Sociales

incondicionalidad y la integración entre el beneficio que se paga y el sistema tributario para garantizar un impacto distributivo progresivo (Lo Vuolo, 2010).

Sería un avance importante el reconocimiento de la ciudadanía como garante de derecho, alejándose del actual sistema en el que el mercado laboral es el mecanismo excluyente de acceso a la protección social. El pleno empleo formal no ha resultado ser la situación "normal" en las economías capitalistas, incluso en las desarrolladas (Beccaria y Maurizio, 2014), por lo que replantear el sistema de protección social ligado al trabajo formal parece ser materia pendiente. El desafío inmediato es ampliar la cobertura para aquellos que se encuentran por fuera de las protecciones del Estado intentando incluir a la mayor parte de la población dentro de los círculos de consumo necesarios para tener una vida digna.

Las propuestas de alguna versión de ingreso básico o ciudadano, si bien no representan una solución definitiva a los problemas sociales de la población, cuestionan algunos principios básicos vinculados al funcionamiento del sistema económico capitalista. Esencialmente, aumenta el poder de negociación de los trabajadores frente a sus potenciales empleadores, dado que -al tener asegurado un mínimo nivel de ingresos que garantiza su reproducción- ya no tendrían que aceptar obligatoriamente cualquier trabajo por necesidad. Por otro lado, esta propuesta también contribuye a la reflexión acerca de qué constituye trabajo realmente útil en nuestra sociedad y nos permitiría pensar en un concepto de trabajo ampliado, en el cual se valoren múltiples actividades y ocupaciones que hoy son consideradas como ocio -el trabajo doméstico y el trabajo comunitario son algunos de ellos-.

La pobreza, el desempleo, la precariedad laboral, son fenómenos plenamente funcionales al sistema de acumulación capitalista $y$, mientras no se cuestionen los pilares básicos sobre los que este se apoya, difícilmente podamos plantear un cambio estructural en el que se promueva una solución verdadera a los problemas que aquejan a nuestras sociedades.

\section{Bibliografía}


Abramovich, V. (2006). Una aproximación al enfoque de derechos en las estrategias y políticas de desarrollo. Revista de La CEPAL, (88), 35-50.

Abramovich, V. y Pautassi, L. C. (2006). Dilemas actuales en la resolución de la pobreza. El aporte del enfoque de derechos. Presented at the "Los Derechos Humanos y las políticas públicas para enfrentar la pobreza y la desigualdad. Buenos Aires: UNESCO, Secretaría de Derechos Humanos y Universidad Nacional Tres de Febrero, p. 40.

Albano, J. y Salas, J. (2007). La inversión en capital humano: sus efectos sobre las retribuciones. En Neffa, J. C.; Albano, J.; López Ghio, R.; Salas, J. y Toledo, F. (eds./comps.)Teorías Económicas sobre el Mercado de Trabajo II. Neoclásicos y nuevos keynesianos .Lugar de edicion: Fondo de Cultura Económica/Trabajo y Sociedad, pp.167-208.

Banco Mundial (2000). Social risk Management: a new conceptual framework for social protection and beyond. Social Protection Discussion paper $N^{\circ} 0006$, World Bank.

Barba Solano, C. y Valencia Lomelí, E. (2011). Hipótesis no comprobadas y espejismos de las transferencias monetarias condicionales. En Barba Solano y Cohen (coords.) Perspectivas críticas sobre la cohesión social. Desigualdad y tentativas fallidas de integración social en América Latina. Buenos Aires: CLACSO.

Barbier, J.C. (2000). A propos des difficultés de traduction des catégories d'analyse des marchés du travail et des politiques de l'emploi en contexte comparatif européen. Documento de trabajo $\mathrm{N}^{\circ}$ 3. lugar de edicion: Centre d'étude de l'emploi (CEE), Universidad de París.

Barbier, J. C. y Knuth, M. (2010). Of similarities and divergences: why there is no continental ideal-type of activation reforms. Lugar de edicion: Centro de Economía de la Sorbonne, Universidad Panthéon-Sorbonne.

Barrientos, A. (2004). Latin America: Towards a liberal-informal welfare regime. En Gough y Wood (eds.) Insecurity and Welfare Regimes in Asia, Africa and Latin America. Social Policy in Development Contexts. Cambridge: University Press.

Barrientos, A. (2011). On the Distributional Implications of Social Protection Reforms in Latin America. Working Papers $N^{\circ}$ 69. UNU-WIDER Research Paper, World Institute for Development Economic Research (UNU-WIDER).

Beccaria, L. y Maurizio, R. (2014). Hacia la Protección social universal en América Latina. Una contribución al debate actual. Revista Problemas Del Desarrollo, 45(177), 37-58.

Becker, G. (1983). El Capital Humano. Madrid: Alianza. 
De Prácticas y discursos/ Universidad Nacional del Nordeste/ Centro de Estudios Sociales

Bonvecchi, A. y Smulovitz, C. (2008). Atender necesidades, crear oportunidades o garantizar derechos. Visiones sobre la política social. En Cruces, G. (comp.) Los Programas Sociales en Argentina hacia el Bicentenario: visiones y perspectivas. Buenos Aires: Banco Mundial.

Castel, R. (2004). La inseguridad social. ¿Qué es estar protegido? Buenos Aires: Manantial.

CEPAL (2007). Las transferencias condicionadas en América Latina: Luces y sombras. Presented at the Seminario Internacional: Programas de transferencias condicionadas: La experiencia de diversos países. Brasilia: CEPAL.

CEPAL (2011a). Programas de transferencias condicionadas: balance de la experiencia reciente en América Latina y el Caribe. Documento de Trabajo $N^{\circ}$ 95. ECLAC: Santiago de Chile.

CEPAL (2011b). Social Panorama of Latin America. Informes Anuales. Santiago de Chile: ECLAC.

Dean, H. (2007). The ethics of welfare-to-work. Policy and Politics, 4(35), 573-590.

Dubet, F. (2010). Repensar la justicia social. Contra el mito de la igualdad de oportunidades. Buenos Aires: Siglo XXI.

Esping-Andersen, G. (1993). Los tres mundos del Estado del bienestar. Valencia: Edicions Alfons el Magnànim-IVEI.

Filgueira, F. (1998). El nuevo modelo de prestaciones sociales en América Latina: residualismo, eficiencia y ciudadanía estratificada. En Roberts, B. R. (ed.) Ciudadanía y Política Sociales. San José de Costa Rica: FLACSO/SSRC, pp. 33-73.

Gautié, J. (2004). Repensar la articulación entre mercado de trabajo y protección social en el posfordismo. Cuadernos de Relaciones Laborales, 22(1), 147-184.

Gazier, B. (1990a). Assurance et chômage, employabilité et marches transitionnels du travail. Université Paris 1/ MATISSE, CNRS UMR 8595, 1-18.

Gazier, B. (1990b). L'employabilité: brève radiographie d'un concept en mutation. Sociologie Du Travail, 32(4), 575-584.

Isuani, A. (2008). La política social argentina en perspectiva. En Cruces, G.; Moreno, J.M.; Ringold, D. y Rofman (eds.) Los programas sociales en Argentina hacia el Bicentenario. Visiones y Perspectivas. Buenos Aires: Banco Mundial, pp. 169-197. 
De Prácticas y discursos/ Universidad Nacional del Nordeste/ Centro de Estudios Sociales

Isuani, A. (2012). Política social y transferencias monetarias en América Latina. Revista de Análisis Público Universidad de Valparaís, 1(1), 14-41.

Landau, M. (2008). Cuestión de ciudadanía, autoridad estatal y participación ciudadana. Revista Mexicana de Sociología, 70(1), 7-45.

Lavinas, L. (2013). 21st century welfare. New Left Review, (84), 5-40.

Lo Vuolo, R. (2009). Asignación por Hijo. Serie de Análisis de Coyuntura, CIEPP, (21), 125.

Lo Vuolo, R. (2010). Las perspectivas del ingreso ciudadano en América Latina. Un análisis en base al "programa Bolsa Familia" de Brasil y a la "Asignación Universal por Hijo" de Argentina. CIEPP.

Morduchowicz, A. (2004). Discusiones de economía de la educación. Buenos Aires: Losada.

Offe, C. (1990). Contradicciones en el Estado de Bienestar. Madrid: Alianza.

OIT (2012). Combatiendo la desigualdad desde lo básico Piso de protección social e igualdad de género. Costa Rica: OIT/PNUD.

OIT (2013). Panorama laboral 2013 en América Latina y el Caribe. Lima: OIT.

OIT (2014). La Estrategia de Desarrollo de los Sistemas de Seguridad Social de la OIT. El Papel de los Pisos de Protección Social en América Latina y el Caribe. Perú: OIT.

Pautassi, L. C. (2010). El aporte del enfoque de Derechos a las políticas sociales Una breve revisión. Presented at the Taller de expertos "Protección social, pobreza y enfoque de derechos: vínculos y tensiones", CEPAL-GTZ, p. 5.

Pérez Eransus, B. (2006). Rentas mínimas y políticas de activación. Documentación Social, (143), 77-92.

Polanyi, K. (2004). La Gran Transformación. Crítica del liberalismo económico. México: La Piqueta.

Procacci, G. (1999). Ciudadanos pobres, la ciudadanía social y la crisis de los Estados de Bienestar. En García, S. y Lukes, S. (eds.) Ciudadanía: justicia social, identidad y participación. Madrid: Siglo XXI.

Rosanvallon, P. (2007). Repensar el Estado Providencia, la nueva cuestión social. Buenos Aires: Manantial.

Rose, N. (1996). The death of the social? Economy and Society, 25(3), 327-356. 
De Prácticas y discursos/ Universidad Nacional del Nordeste/ Centro de Estudios Sociales

Salama, P. (2011). Luchas contra la pobreza en América Latina. El caso de la pobreza rural en Brasil. Revista Problemas Del Desarrollo, 165(42), 7-34.

Schultz, T. W. (1961). Investment in Human Capital. The American Economic Review, 51(1), 1-17.

Skoufias, E. (2000). ¿Está dando buenos resultados Progresa? Documento de trabajo de IFPRI - SEDESOL, México.

Stampini, M.y Tornarolli, L. (2012). The Growth of Conditional Cash Transfers in Latin America and the Caribbean: Did They Go Too Far? IDB Policy Brief.

Valencia Lomelí, E. (2008). Las transferencias monetarias condicionadas como política social en América Latina. Un balance: aportes, límites y debates. Annual Review of Sociology, (34), 499-524. 